\title{
MFI Based Interoperability Measurement of Business Models in Service-based Enterprises
}

\author{
Zhao Li \\ College of Computer and Information Technology \\ China Three Gorges University, Yichang, China \\ zhaolicst@163.com
}

\begin{abstract}
Various business models of service-based enterprise exist and are currently used in industry, whilst their definitions, structures, functions, and supporting tools are quite different from each other. For interoperability, the partial semantic interoperability between heterogeneous business models is challenging to achieve. Almost all of the enterprise business models can be described from the four major dimensions: Role, Goal, Process, Service (RGPS), consequently in this paper, a business model of service-based enterprise is actually a specific RGPS model, an approach for measuring the interoperability of RGPS models is proposed. At first, the RGPS interoperability features framework is constructed based on Meta-model Framework of Interoperability (MFI), and it is specified to be the interoperability features set of RGPS models; secondly, the interoperability features set and a mathematical method are proposed to identify and quantify a RGPS model and its interoperability features, then the model instance of the RGPS model is produced; next, we calculate the similarity between two model instances, and obtain the measuring results of interoperability between corresponding RGPS models, which is used to build the interoperability measurement matrix of RGPS models set.
\end{abstract}

Keywords-interoperability measurement; MFI; heterogeneous business models; service-based enterprise; interoperability features

\section{INTRODUCTION}

Different organizations and institutions define the term "interoperability" differently. The definition with high citation count in Google Scholar [1] indicates interoperability is the ability of two or more software components to cooperate despite differences in language, interface, and execution platform. IEEE standard computer dictionary [2] defines interoperability as the capability of two or more systems or components to exchange information and to use the information that has been exchanged, this definition includes two meanings: the ability of both ends to exchange information, that is a technology interoperability; the ability of receiving end to utilize the information exchanged, it is a semantic interoperability. In this paper, the definition of interoperability given by IEEE standard computer dictionary is adopted.

Interoperability is multidimensional and very complex to assure. It is difficult and also essential to accurately measure and report the specific interoperability [3]. Currently, the study scope of interoperability primarily focuses on the definition of interoperability, the interoperability of the data layer as well as the metadata layer, such as Levels of Information System Interoperability (LISI) [4], which is the earliest interoperability measurement based on a maturity

\author{
Peng Liang, Yi Zhao, Keqing He \\ State Key Lab of Software Engineering \\ Wuhan University, Wuhan, China \\ liangp@sklse.org; ivwepriu@sina.com; \\ hekeqing@whu.edu.cn
}

model. LISI defines the measurable levels of interoperability by setting corresponding interoperability thresholds. LISI consists of five levels (from Level 0 to Level 4), Level 0 is Isolated and Level 1 is Connected, both of which identify the weaker interoperability, and the interoperability attributes involved is about the data layer. Level 2 (Functional), Level 3 (Domain), and Level 4 (Enterprise) identify the stronger interoperability, and the interoperability attributes involved is integrated from the metadata layer; Office of the Under Secretary of Defense [5] points out that in the interfaces management of Systems of Systems, a single system is a certain part of the entire SoS, so it will affect the entire SoS once some interfaces of a single system change, for this, the key issues need to be addressed is the effective integration of data and metadata, as well as the pre-registration and management of metadata within the related resources. However, there is still a large space to study the partial semantic interoperability and interoperability measurement of model layer.

The rest of this paper is organized as follows. In Section II, we discuss related work about interoperability measurement, and model interoperability. Section III presents our approach for measuring interoperability between RGPS models. We conclude our work and discuss the future work in Section IV.

\section{RELATED WORK}

In the past few decades, the studies of interoperability and interoperability measurement have been extensively concerned in industry. Along with a large number of emergences of research and the continuing rapid development of technology, the study topic of interoperability will become more important in all walks of life, especially in the field of ICT. Amanowicz et al [6] specify that interoperability locates at the higher layer of communication and information system standard, which is the key to achieve the collaboration between each layers of heterogeneous communication systems. Leite's work [7] indicates that in order to measure the interoperability between systems more accurately and completely, the mathematical method must be adopted to quantify the interoperability components. When various military organizations take joint actions, the ability and degree of collaboration among them is the key to take the successful actions. So Clark et al [8] point out that only if both parties sufficiently understand the interoperability of the organizations, they can command and control the military organizations participate in the joint actions.

Over the past decade, a large number of studies about "model interoperability" have emerged in industry. In this 
paper, we use online electronic database ScienceDirect, IEEE Xplore, Springer Link, and ACM Digital Library to retrieve the keyword "model interoperability" in title item, thus then we obtain the statistics of the related academic papers in the past ten years, as shown in Figure 1. In view of the statistics, to some extent, we illustrate the research trends about model interoperability, as shown in Figure 2.

Figure 2 shows in academia and industry, the research on model interoperability presents a trend of steadily increasing year by year since 2002, which indicates that the research on model interoperability will be paid close attention to in the future, and the related works will also permeate into more domains.

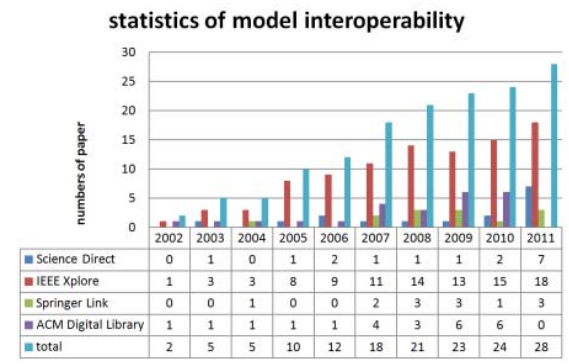

Figure 1. The paper statistics of model interoperability.

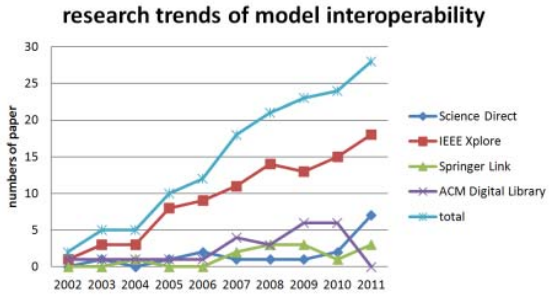

Figure 2. The research trends about model interoperability.

In view of above contents, the effective implementation of interoperability and interoperability measurement on the model layer is still difficult to achieve, especially reflected in the follow aspects:

- The categories and classification of the existing interoperability measurement are confusion.

- The study scope of interoperability measurement is often limited to the data layer as well as the metadata layer.

- Each approach is only adequate for the specific application environment, the versatility of which is less.

- The studies of collaborative interoperability between models usually lay particular emphasis on roughly qualitative analysis, which cause the low accuracy of interoperability measurement between models.

\section{INTEROPERABILITY MEASUREMENT OF RGPS MODELS}

In this section we combine the numerical taxonomy [9] with data similarity computing technique [10] to explore the features states of a specific RGPS (Role Goal Process Service) model quantitatively, and an approach for measuring the interoperability between RGPS models is proposed. Our approach is based on the four interoperability frameworks [11] [12] [13] contained in MFI (Meta-model Framework of Interoperability), which are developed by the authors.

Firstly, we construct the RGPS interoperability features framework based on MFI, and define it to be the interoperability features set of RGPS models. At second, the interoperability features set and a mathematical method are adopted to identify and quantify a RGPS model and its interoperability features, thus then the corresponding model instance is produced. Next, we calculate the similarity between two model instances, and obtain the interoperability between corresponding RGPS models, which is used to build the interoperability measurement matrix of RGPS models set. Finally, the steps for interoperability measurement of RGPS models are presented. Our overall approach is illustrated in Figure 3.

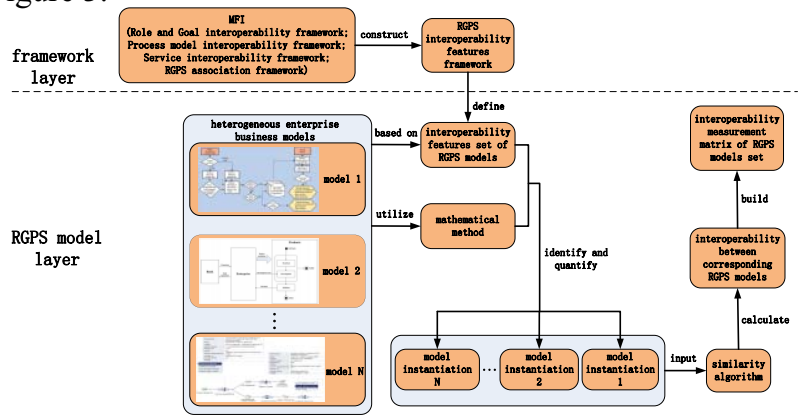

Figure 3. Overall approach for interoperability measurement of RGPS models.

\section{A. Foundation}

The four interoperability frameworks contained in MFI are Role and Goal interoperability framework [11], Process model interoperability framework [11], Service interoperability framework [12], and RGPS association framework [13]. Role and Goal interoperability framework is primarily used to support interoperability registration and management of role and goal models, and define two elements needed for the interoperability between business models of service-based enterprises, namely the "Role" and "Goal", as well as the related expanding elements; Process model interoperability framework aims at supporting interoperability registration and management of process models, and define the element "Process" and the related expanding elements needed for the interoperability between business models; Service interoperability framework provides supports for interoperability registration and management of service models, as the same, define the element "Service" and the related expanding elements; and RGPS association framework is responsible for elaborating the relationships between the first three frameworks, that is to say how could these elements associate with each other and combined as a whole.

Numerical taxonomy is first proposed by Sneath et al, in their work, the concept of numerical taxonomy is specified: utilizing numerical method and the features states of objects to classify the objects [9]. Numerical taxonomy is very 
versatile, so it is widely used in many fields of science research, such as biology, medicine, chemistry, information science, and social science. In some disciplines, numerical taxonomy is the theoretical basis of such discipline.

In order to measure the interoperability between business models of service-based enterprise (specific RGPS models), we first need to calculate the similarity between business models. We adopt the concepts of feature and feature state in numerical taxonomy to describe the specific business models, thus then the data about the features of related models is produced. We can transform the similarity computing between business models into the similarity computing between the features of different models. In application, the business models are very heterogeneous from each other, for example, the categories of business models are plentiful, the structures are different, the business scope are complex, and the description languages are limited into a single application, which cause that the data about the features of each model are very heterogeneous from each other, and it is difficult to execute quantitative analysis for the features. To quantify the features states of business models, and provide comprehensive supports for the interoperability measurement of heterogeneous RGPS models, the RGPS interoperability features framework and the interoperability features set of RGPS models are both need.

\section{B. Interoperability Features Framework and Features Set}

We empirically cut out the elements of abstract metaclass layer in the four interoperability frameworks contained in MFI, as well as delete the elements not directly involved in the business constructing. Finally, the RGPS interoperability features framework is produced according to the purpose of interoperability measurement, as shown in Figure 4.

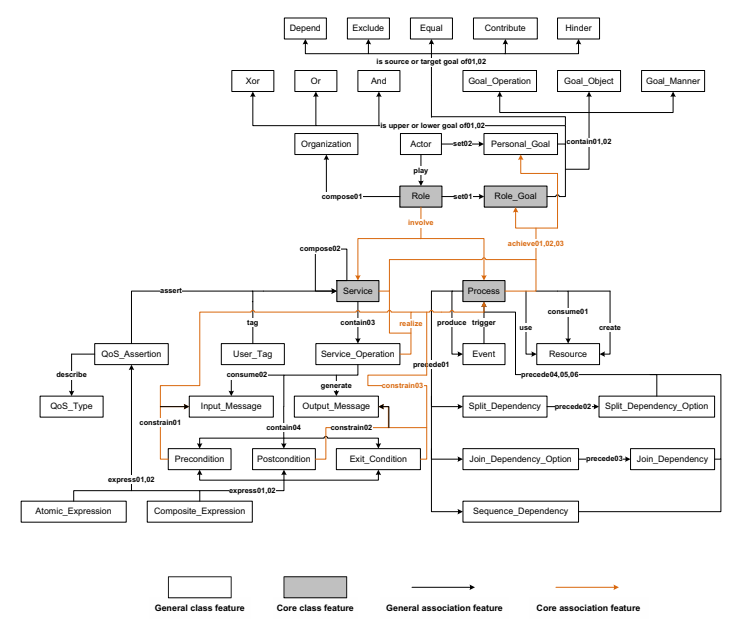

Figure 4. RGPS interoperability features framework.

RGPS interoperability features framework consists of 36 class features and 39 association features. Four of them are Core class feature: Role, Role_Goal, Process, and Service, which are used to specify the scope described by each. As the same, eight of these features are Core association feature: involve, achieve01, achieve02, achieve03, realize, constrain01, constrain02, and constrain03, which describe the associations between the scopes. Since each class feature and its out-degree association feature (the arrow starts from a class feature and points outside) form a whole, for example, the out-degree association features of Role are compose 01 , involve, and set01, so each class feature and its out-degree association features are dealt with as a whole, TABLE I sequences these interoperability features of RGPS models.

TABLE I. SEQUENCING TABLE OF INTEROPERABILITy FEATURES

\begin{tabular}{|c|c|c|}
\hline No. & class feature & association feature \\
\hline 1 & Role & 1.1.1set01/1.2involve/1.3compose01/ \\
\hline 2 & Actor & 2.1 play $/ 2.2$ set 02 \\
\hline 3 & Organization & \\
\hline 4 & Role_Goal & 4.1 contain $01 / 4.2$ is upper or lower goal of $01 / 4.3$ is source or target goal of 01 \\
\hline 5 & Personal Goal & 5.1 contain $02 / 5.2$ is upper or lower goal of $02 / 5.3$ is source or target goal of 02 \\
\hline 6 & Depend & \\
\hline 7 & Exclude & \\
\hline 8 & Equal & \\
\hline 9 & Contribute & \\
\hline 10 & Hinder & \\
\hline 11 & And & \\
\hline 12 & Or & \\
\hline 13 & Xor & \\
\hline 14 & Goal_Operation & \\
\hline 15 & Goal_Object & \\
\hline 16 & Goal Manner & \\
\hline 17 & Process & 17.1 precede $01 / 17.2$ achieve $01 / 17.3$ produce $/ 17.4$ use $/ 17.5$ consume $1 / 17.6$ create \\
\hline 18 & Event & 18.1trigger \\
\hline 19 & Resource & \\
\hline 20 & Split_Dependency & 20.1 precede 02 \\
\hline 21 & Join Dependency & 21.1 precede 06 \\
\hline 22 & Sequence_Dependency & 22.1 precede 04 \\
\hline 23 & Split_Dependency_Option & 23.1 precede 05 \\
\hline 24 & Join_Dependency_Option & 24.1 precede 03 \\
\hline 25 & Service & 25.1 1achieve $02 / 25.2$ compose $02 / 25.3$ contain 03 \\
\hline 26 & Service_Operation & 26.1achieve $03 / 26.2$ realize $/ 26.3$ consume $02 / 26.4$ contain $04 / 26.5$ generate \\
\hline 27 & User_Tag & 27.1tag \\
\hline 28 & QoS Assertion & 28.1assert 28.2 describe \\
\hline 29 & QoS Type & \\
\hline 30 & Input_Message & \\
\hline 31 & Output_Message & \\
\hline 32 & Precondition & 32.1 constrain 01 \\
\hline 33 & Postcondition & 33.1constrain 02 \\
\hline 34 & Exit_Condition & 34.1constrain 03 \\
\hline 35 & Atomic_Expression & 35.1 express0I \\
\hline 36 & Composite Expression & 36.1 express 02 \\
\hline
\end{tabular}

These interoperability features of RGPS models are sequenced strictly, in order to keep the mathematical method more simple, and promote the identification and quantification of models and features, in this paper, the fixed sequence specified in TABLE I must be strictly complied with. That is, the No.1 feature Role is first identified, and then 1.1set is second identified, next, 1.2involve is third identified, thus then 1.3compose01 is identified, and then 2Actor is identified, and so on, finally the course is ended with the identification of 36.1express02. The sequenced interoperability features set of RGPS models avoids bringing a lot of obstacles for interoperability measurement, and provides supports for the identification and quantification of RGPS models and their interoperability features.

\section{Identification and Quantification of Models and Features}

To accurately identify and quantify RGPS models and their interoperability features, as well as calculate the similarity between model instances effectively, the analysis for the type and nature of the interoperability features is essential, which is also the premise to identify and quantify model features mathematically.

According to the elaborations of nominal attribute and binary attribute given by Han et al [10], we can see that the interoperability features of RGPS models are nominal features, the descriptions of business models are achieved 
through the representations of these interoperability features. For the representations of interoperability features, we regard them to be equivalent to whether a business model contains these interoperability features, further regard the interoperability features of RGPS models as the binary features with a discrete state 0 or 1 , where the state 0 indicates that a business model doesn't contain this feature, on the contrary, the state 1 indicates a business model indeed contain the feature. The states of 0 and 1 are not equally important for the interoperability between models, that is to say, the feature state 1 is more valuable for the interoperability measurement between models, so we treat the nominal interoperability features to be the asymmetric binary features [10], finally the interoperability measurement of RGPS models is simplified and promoted.

After the transformation from nominal features to asymmetric binary features, the corresponding mathematical method is developed in this section to identify and quantify the RGPS models and related interoperability features. For various business models of service-based enterprises, RGPS interoperability features set is not only able to accurately describe and distinguish different business models according to the four scopes: Role, Role Goal, Process, and Service, but also is the minimum features set for effectively measuring the interoperability between business models.

In the course of identifying and quantifying the interoperability features, we develop a mathematical method consisting of two definitions:

Definition 1: Mapping from RGPS models to interoperability features.

$$
I F: R G P S M \rightarrow F S
$$

RGPSM represents the set of RGPS models, and $R G P S M=\left\{m_{(i)}|i=1,2, \ldots| R G P S M \mid,\right\}, m_{(i)}$ represents the $i$-th model in $R G P S M$; IF represents the interoperability features set of RGPS models, and is used to describe RGPSM, $I F=\left\{f_{(k)}|k=1,2, \ldots| I F \mid,\right\}, f_{(k)}$ is the $k$-th interoperability feature, such as $f_{(1)}$ represents the feature Role, and in this paper, $I F$ is:

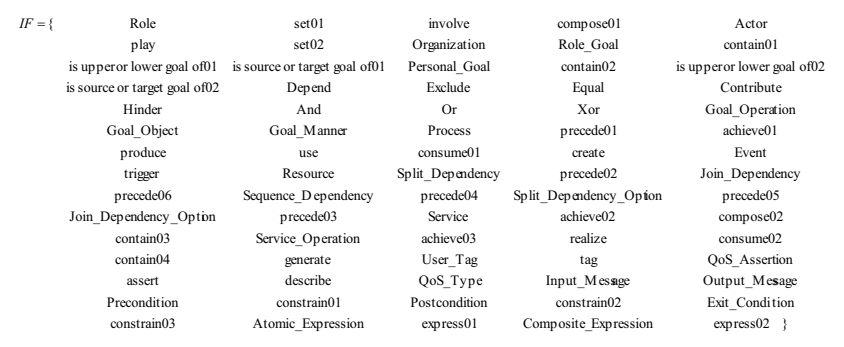

The sequence of the interoperability features in $I F$ strictly complies with the fixed sequence specified in TABLE I; and $F S$ is the interoperability features states set of RGPS models, and is used to describe all possible model features states, $F S=\{0,1\}$, where 0 indicates that a RGPS model doesn't contain this feature, on the contrary, 1 indicates a RGPS model indeed contain the feature. So the purpose of function (1) is to achieve the mapping from RGPS models to the interoperability features states. Definition 1 is referenced from Semple et al [14].

Definition 2: Model instantiation.

$$
\alpha=I F(m), m \in R G P S M
$$

$$
\operatorname{MIM}=\left[\alpha_{(i)}\right]=\left[\operatorname{IF}\left(m_{(i)}\right)\right], i=1,2, \ldots,|R G P S M|
$$

In function (2), $m$ is a specific RGPS model and belongs to RGPSM. The course of using $I F$ to describe a specific model $m$ is equivalent to the instantiation of model $m$, after that, the interoperability features states sequence of model $m$ is produced, and we name it as $\alpha$, which is also known as the instance of $m$. The features states $\{0,1\}$ in $\alpha$ are strictly sequenced following the fixed sequence specified in $I F$. So the function (2) is used to instantiate a specific model; in function (3), $\alpha_{(i)}$ is the instance of $m_{(i)}$, and $i=1,2, \ldots,|R G P S M|$. $M I M$ represents the model instance matrix of $R G P S M$, that is to say, if $\forall m_{(i)} \in R G P S M, \quad \exists \alpha_{(i)} \in M I M$, and $M I M=\left\{\alpha_{(i)}|i=1,2, \ldots| R G P S M \mid,\right\}$, so we treat $M I M$ as the instantiation of RGPSM.

Definition 1 and 2 together constitute the mathematical method proposed in this section.

\section{Interoperability Computing of RGPS Models}

The interoperability between two RGPS models can be represented by the similarity computing between two model instances. Since the asymmetric binary feature is utilized in the identification and quantification of interoperability features, the Jaccard coefficient [10] is referenced to calculate the similarity between the model instances, which is well suited for the interoperability measurement with asymmetric binary features. Once the two model instances are produced, we can calculate the similarity of them in function (4), which also represents the outcome of interoperability between the corresponding RGPS models.

$$
M I_{\left(m_{(i)}, m_{(j)}\right)}=M S_{\left(\alpha_{(i)}, \alpha_{(j)}\right)}=\frac{\sum_{k=1}^{|I F|} C_{\left(\alpha_{(i)}, \alpha_{(j)}\right)}^{\left(f_{(k)}\right)} \cdot W_{\left(\alpha_{(i)}, \alpha_{(j)}\right)}^{\left(f_{(k)}\right)}}{\sum_{k=1}^{|I F|} C_{\left(\alpha_{(i)}, \alpha_{(j)}\right)}^{\left(f_{(k)}\right)}}
$$

Where $\alpha_{(i)}$ and $\alpha_{(j)}$ are respectively the instances of model $m_{(i)}$ and $m_{(j)}$, and $m_{(i)}, m_{(j)} \in R G P S M, M I_{\left(m_{(i)}, m_{(j)}\right)}$ represents the interoperability between model $m_{(i)}$ and $m_{(j)}, M S_{\left(\alpha_{(i)}, \alpha_{(j)}\right)}$ is the similarity of instance $\alpha_{(i)}$ and $\alpha_{(j)}$, and $f_{(k)}$ is the $k$-th interoperability feature, $I F=\left\{f_{(k)}|k=1,2, \ldots| I F \mid,\right\}$; On the one hand, $C_{\left(\alpha_{(i)}, \alpha_{(j)}\right)}^{\left(f_{(k)}\right)}$ represents the distinguisher used to distinguish whether the states of the $k$-th interoperability feature in $m_{(i)}$ and $m_{(j)}$ simultaneously equal to 0 , that is to say, $f_{\left(k, m_{(i)}\right)}$ and $f_{\left(k, m_{(j)}\right)}$ are respectively the $k$-th 
interoperability feature of $m_{(i)}$ and $m_{(j)}$, if $f_{\left(k, m_{(i)}\right)}=f_{\left(k, m_{(j)}\right)}=0$, distinguisher $C_{\left(\alpha_{(i)}, \alpha_{(j)}\right)}^{\left(f_{(k)}\right)}=0$, otherwise $C_{\left(\alpha_{(i)}, \alpha_{(j)}\right)}^{\left(f_{(k)}\right)}=1 \quad ; \quad$ On the other hand, $W_{\left(\alpha_{(i)}, \alpha_{(j)}\right)}^{\left(f_{(k)}\right)}$ represents the weight used to judge whether the states of the $k$-th interoperability feature in $m_{(i)}$ and $m_{(j)}$ are equivalent, in other words, if $f_{\left(k, m_{(i)}\right)}=f_{\left(k, m_{(j)}\right)}$, weight $W_{\left(\alpha_{(i)}, \alpha_{(j)}\right)}^{\left(f_{(k)}\right)}=1$, otherwise $W_{\left(\alpha_{(i)}, \alpha_{(j)}\right)}^{\left(f_{(k)}\right)}=0$. Through the distinguisher and the weight, we can not only ignore the feature of both states 0 in model $m_{(i)}$ and $m_{(j)}$, but also exclude the feature of different states. Finally, the outcome of interoperability between two RGPS models is produced.

According to function (4), we can calculate the interoperability between any two models in RGPSM to construct the interoperability measurement matrix of RGPSM, which is represented as IMM in function (5).

$$
\begin{gathered}
I M M=\left[M_{\left(m_{(i)}, m_{(j)}\right)}\right], m_{(i)}, m_{(j)} \in R G P S M \\
i, j=1,2, \ldots|R G P S M|
\end{gathered}
$$

\section{E. Steps for Interoperability Measurement}

The interoperability measurement of RGPS models in this paper can be illustrated in the following three steps:

- $\quad$ Step 1)-identify and quantify the RGPS models and their interoperability features: we utilize IF specified in TABLE I and function (1), (2), (3) to identify the RGPS models and quantify the

\section{REFERENCES}

[1] P. Wegner, "Interoperability," ACM Computing Surveys (CSUR), vol. 28, pp. 285-287, 1996.

[2] IEEE, IEEE standard computer dictionary: a compilation of IEEE standard computer glossaries. New York: Institute of Electrical and Electronics Engineers, 1990.

[3] M Kasunic, and W Anderson, Measuring Systems Interoperability: Challenges and Opportunities, CMU/SEI-2004-TN-003. Pittsburgh, PA: Carnegie Mellon University Software Engineering Institute, 2004.

[4] Department of Defense, C4ISR Architecture Working Group Final Report - Levels of Information System Interoperability. Washington DC: OSD(ASD(C3I)) C4ISR AWG, 1998.

[5] Office of the Deputy Under Secretary of Defense for Acquisition and Technology-Systems and Software Engineering, Systems Engineering Guide for Systems of Systems, Version 1.0. Washington DC: ODUSD(A\&T)SSE, 2008.

[6] M. Amanowicz, and P. Gajewski, "Military Communications and Information Systems Interoperability," in Proceedings of the 1996 IEEE Military Communications Conference, pp. 280-283, 1996. interoperability features, then output RGPSM and MIM.

- $\quad$ Step 2)-calculate the interoperability between any two models in the RGPS models set: we input RGPSM and MIM into function (4) to calculate the outcome of interoperability between any two models in $R G P S M$, next output each MI.

- Step 3)-construct the interoperability measurement matrix: we input each $M I$ into function (5), and output the corresponding IMM.

\section{CONCLUSION}

It is challenging to study and assure the interoperability of service-based enterprise at the model layer. In this paper, we propose an approach based on MFI to measure the interoperability of heterogeneous RGPS models, which consists of three steps: identify and quantify the RGPS models and their interoperability features; calculate the interoperability between any two models in the RGPS models set; and construct corresponding interoperability measurement matrix. The major contributions of this work are:

- Using the four dimensions (RGPS) of MFI as the foundation to enhance the integrality of interoperability measurement between business models.

- Combining qualitative analysis and quantitative calculation, which improving the accuracy of interoperability measurement.

\section{ACKNOWLEDGMENT}

This work was supported by the National Science and Technology Pillar Program of China [2012BAH07B01]; and the National Nature Science Foundation of China [60970017].

[7] M. Leite, "Interoperability Assessment," in Proceedings of the 66th Military Operations Research Society Symposium, Monterey, CA, 1998.

[8] T. Clark, and R. Jones, "Organisational Interoperability Maturity Model for C2," in Proceedings of the 9th International Command and Control Research and Technology Symposium, Newport, RI, 1999.

[9] P. Sneath, and R. Sokal, Numerical Taxonomy, San Francisco, CA: W. H. Freeman and Company, 1973.

[10] J. W. Han, M. Kamber, and J. Pei, Data Mining: Concepts and Techniques, 3rd ed., Waltham, MA: Elsevier Inc, 2012.

[11] Z. Li, Z. Li, and H. T. Li et al, "Formalization of Rules for the Cooperation between Goal and Process," Journal of Donghua University, vol. 29, pp. 32-36, 2012.

[12] Z. W. Feng, R. Peng, and B. Li et al, "A Service Registry Meta-model Framework for Interoperability," in Proceedings of the 10th International Symposium on Autonomous Decentralized Systems (ISADS), Tokyo, Hiroshima,pp. 389-398, 2011.

[13] J. Wang, "Research on Requirements Meta-modeling Framework and Key Techniques of Networked Software," Ph.D. dissertation, Dept. Computer School., WuHan Univ., Wuhan, MS, 2008.

[14] Semple, and M. Steel, Phylogenetics, Oxford: Oxford University Press, 2003. 